\title{
A Biofilm Growth Protocol and the Design of a Magnetic Field Exposure Setup to Be Used in the Study of Magnetic Fields as a Means of Controlling Bacterial Biofilms
}

\author{
Bruce R. McLeod ${ }^{1,2 *}$ and Elizabeth L. Sandvik ${ }^{2,3}$ \\ ${ }^{1}$ Department of Electrical and Computer Engineering, Montana State University, \\ Bozeman, Montana \\ ${ }^{2}$ Center for Biofilm Engineering, Montana State University, Bozeman, Montana \\ ${ }^{3}$ Chemical \& Biological Engineering, Montana State University, Bozeman, Montana
}

\begin{abstract}
The use of prosthetic implants is increasing both in the United States and around the world and there is a concomitant rise in cases of biofilm-based, persistent infections that are quite serious and virtually impervious to antibiotic treatment. The development of alternate therapies that do not involve long term use of high levels of antibiotics or surgical intervention is needed. Based on the success of using electric or magnetic fields to alter certain physiological processes, it is hypothesized that relatively low level magnetic fields, in conjunction with the appropriate antibiotic, may be able to help control and eventually clear bacterial biofilms on a prosthetic. In order to test this hypothesis, it is necessary to first develop a means of growing laboratory grade biofilms on specific materials in a way that is repeatable between experiments and that can be reproduced by other laboratories. Secondly, a means of applying controlled magnetic fields to the surfaces supporting the biofilms at a defined temperature must be developed. This article addresses both of these points. Bioelectromagnetics 31:56-63, 2010. @ @ 2009 Wiley-Liss, Inc.
\end{abstract}

Key words: Staphylococcus epidermidis; prosthetic implants; orthopedic device associated infections; EMF; solenoid

\section{INTRODUCTION}

Bacterial biofilm growth on a prosthetic implant can be the primary cause of persistent infections resulting in serious medical problems [König et al., 2001; Darouiche, 2004; Pavoni et al., 2004; Saginur et al., 2006]. Bacterial biofilms have a well-known ability to be nearly impervious to antibiotic treatment, with resistance to antibiotics at 1000 to as high as 5000 times the concentration effective on the same species, in a planktonic (free-floating) culture [Costerton and Stewart, 2000; Del Pozo et al., 2008]. Extensive antimicrobial therapy with multiple antibiotics has been used as a treatment for implantassociated infections [Zimmerli et al., 1998; König et al., 2001; Pavoni et al., 2004] but if this course of action fails, it is probable that the removal and replacement of the device in a one- or two-stage surgical process, in addition to another extensive course of antibiotics, will be necessary [Pavoni et al., 2004]. An effective treatment method that improves antibiotic treatment and does not include surgery is clearly needed.
One alternate approach to traditional antimicrobial treatment is the use of electric or magnetic fields alone, or in conjunction with an appropriate antibiotic, to enhance the efficacy of the antibiotic against the biofilm. A 1992 study first showed that the action of several specific biocides against Pseudomonas aeruginosa biofilms could be significantly enhanced with the application of low levels of direct current (DC) whereas the biocide or current alone had little to no

Grant sponsors: Peter Ewing Capital Management LLC; Allegheny-Singer Research Institute; Montana Board of Research and Commercialization Technology (08-03).

*Correspondence to: Bruce R. McLeod, Professor of Electrical Engineering, Room 619 Cobleigh Hall, Montana State University, Bozeman, MT 59717-2580. E-mail: mcleod@montana.edu

Received for review 22 September 2008; Final revision received 18 May 2009

DOI 10.1002/bem.20529

Published online 23 July 2009 in Wiley InterScience (www.interscience.wiley.com). 
effect [Blenkinsopp et al., 1992]. A number of similar articles have since appeared [Wellman et al., 1996; Masterson et al., 1998; Stewart et al., 1999; O'Gara and Humphreys, 2001; Caubet et al., 2004; Ehrlich et al., 2005; Shirtliff et al., 2005; Del Pozo et al., 2008] indicating the effectiveness of this direct current approach. While this approach may show promise in clinical treatment, it requires the placement of two electrodes in contact with the patient.

A recent article in Antimicrobial Agents and Chemotherapy suggests AC electric fields alone can inhibit the growth of planktonic bacteria [Giladi et al., 2008]. Time-varying (AC) and non-time varying (DC) electric and magnetic fields have been investigated for quite some time, with respect to their use in medicine and biology (see, e.g., Blank, 1993) and their clinical use in aiding bone repair is well established [Aaron et al., 2004; Pilla et al., 2006]. There are, however, very few reports of using $\mathrm{AC}$ or combined $\mathrm{AC}$ and $\mathrm{DC}$ magnetic fields, in conjunction with antibiotics, to explore the possibility of controlling a bacterial biofilm. A potential advantage of using AC magnetic fields rather than direct current to achieve the control is that no direct contact to the patient is required making this method entirely noninvasive.

In order to explore this approach, a growth protocol for biofilms is needed that allows the biofilms to be grown in a controlled, repeatable manner on specific surfaces and then exposed to well-characterized and defined magnetic fields. Additionally, the entire process must take place at a set temperature. This article first details an approach for growing Staphylococcus epidermidis biofilms on selected materials, and then a magnetic field exposure system design is described that allows for up to seven variations of biofilm experimental conditions (e.g., antibiotic level, antibiotic type, trace minerals, nutrients, type of material, specific biofilm) to be studied during a single experiment, done in a uniform magnetic field at a selected temperature.

\section{BIOFILM GROWTH AND QUANTIFICATION}

The first challenge in biofilm research is to develop a protocol that results in a routinely repeatable biofilm on a desired material. An example of such research would be growing S. epidermidis or Staphylococcus aureus biofilms on materials normally used in prosthetic knees (such as ASTM-75 metal or Ultra High Molecular Weight Polyethylene (UHMWPE)). The requirement for repeatability must hold between experiments and between separate laboratories. The Center for Biofilm Engineering (CBE) at Montana State University has developed a number of growth methods, protocols, and reactors. It should be noted that reactor choice is critical since the growth protocol can affect observed antimicrobial response even when the same microorganism is used. A recent study investigating fluid dynamics as a critical control parameter, looked at two different bacterial species grown in five different growth systems used to study biofilms, and the response to three different antimicrobials. Even though treatments were applied in exactly the same way, the log reduction for the same antimicrobial at the same concentration was significantly different between the different growth systems [Goeres et al., 2005; Buckingham-Meyer et al., 2007].

The CDC Biofilm Reactor (BioSurfaces Technologies, Bozeman, MT) is a continuous flow system with biofilms grown under high shear (shown in Fig. 1a). Figure $1 \mathrm{~b}$ shows the CDC Reactor in use in a typical growth setup. The CDC Reactor is an excellent choice for growing biofilms because of its versatility (it can be used for many different organisms grown on different types of material) and because it can produce biofilms on up to 24 sampling surfaces during a single growth procedure. An example growth protocol for $S$ epidermidis ATCC \#35984 using this reactor follows, which has yielded biofilms with repeatable cell densities $\left( \pm 0.25 \log \mathrm{CFU} / \mathrm{cm}^{2}\right)$ both within and between experiments. While this protocol can be used as a basic guide, some modifications may be needed if a different strain or species of microorganism is to be studied. Additionally, a biofilm sampling process is described. This section was included to indicate how one might evaluate the effect on the biofilm after a magnetic field experiment, but it should be noted that several other sampling processes might work equally well. Both the growth and sampling protocols parallel the methods of ASTM Method E 2562-07 for Pseudomonas aeruginosa biofilms, grown with high shear and continuous flow using the CDC Biofilm Reactor [ASTM International, 2007].

\section{Biofilm Growth Protocol}

S. epidermidis ATCC\#35984 biofilms were grown on polycarbonate coupons $(12.7 \mathrm{~mm}$ diameter, surface area $126.7 \mathrm{~mm}^{2}$ ) in the CDC Biofilm Reactor. The sterilized reactor containing $450 \mathrm{ml}$ of full strength tryptic soy broth (TSB), (Fischer Scientific \#DF0370075, Atlanta, GA) with the effluent tube clamped, was inoculated from a frozen stock of S. epidermidis. The reactor was placed in a $37^{\circ} \mathrm{C}$ incubator and operated in batches while stirring at $125 \mathrm{rpm}$ for $24 \mathrm{~h}$. After $24 \mathrm{~h}$, a continuous flow of sterile 1/10th strength TSB was started with a reactor residence time of $30 \mathrm{~min}$. Continuous flow ran for $16 \mathrm{~h}$ and at the end biofilm-coated coupons were ready for use in experimental protocols. It should be noted that 
the two faces of the coupon (facing inward toward the baffled stir bar versus outward toward the glass) experience different amounts of shear stress which is a critical control in biofilm growth. The inward facing side of the coupon was always of interest for these experiments.

\section{Biofilm Sampling Protocol}

Growth control coupons sampled at the end of the growth protocol were aseptically removed from the rod,

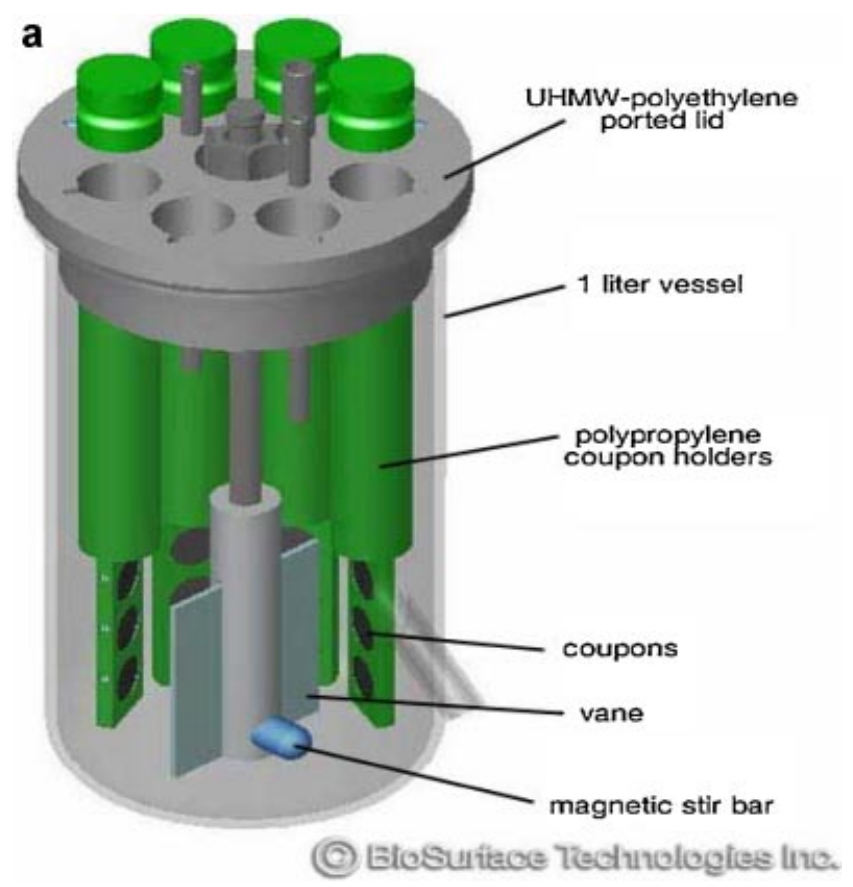

b

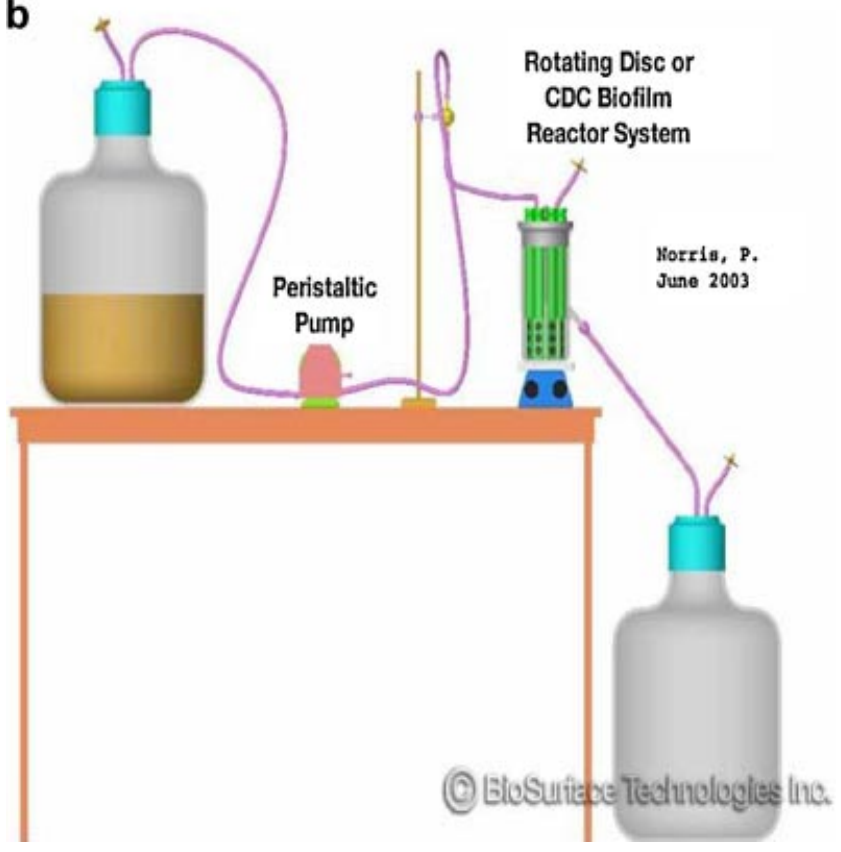

gently dipped in $10 \mathrm{ml}$ of sterile dilution water $(42.2 \mathrm{mg}$ $\mathrm{KH}_{2} \mathrm{PO}_{4} / \mathrm{L}, 406.5 \mathrm{mg} / \mathrm{L} \mathrm{MgCl}_{2} \cdot 6 \mathrm{H}_{2} \mathrm{O} / \mathrm{L}$ in reagentgrade water) to remove planktonic cells, and placed on a sterile Plexiglas board for sampling, with the inward side facing up. The top of the coupon was scraped for $30 \mathrm{~s}$ with a sterile wooden stick held perpendicular to the surface and then rinsed by swirling in $9 \mathrm{ml}$ of dilution water. This process was repeated so that the coupon was scraped three times. After scraping, the coupon was held over the dilution tube and the scraped surface was rinsed with $1 \mathrm{ml}$ of dilution water to remove any remaining biofilm resulting in a final volume of $10 \mathrm{ml}$ in the dilution tube. The sample was homogenized at $10000 \mathrm{rpm}$ for $1 \mathrm{~min}$ to disaggregate biofilm clumps, serially diluted, and drop plated on tryptic soy agar (Fischer Scientific \#DF0369078). After overnight incubation at $37^{\circ} \mathrm{C}$, the colony-forming units (CFU) on the plates were counted and a biofilm density (mean colony-forming units per $\mathrm{cm}^{2}$ ) for each coupon was calculated using the surface area of the coupon. Using the above protocol, a mean cell density for 13 separate experiments was $8.37 \pm 0.25 \log \mathrm{CFU} / \mathrm{cm}^{2}$. Coupons (in place in the rods) used in experimental protocols were dipped to remove planktonic cells upon removal from the CDC Reactor, just prior to transfer to the exposure setup. Following exposure, coupons were removed from the rods and scraped as described above.

\section{MAGNETIC FIELD EXPOSURE SETUP}

Design requirements for the magnetic field exposure setup included being able to achieve a constant elevated temperature (above room temperature), developing and maintaining a controlled, uniform magnetic field, and having multiple experimental conditions tested simultaneously along with a control. Inside a typical laboratory incubator, the AC magnetic

Fig. 1. a: The CDC reactor. Biofilms are grown on $12.7 \mathrm{~mm}$ diameter disks, or coupons, suspended in the bulk fluid by eight polypropylene coupon holders with the possibility of 24 repeat coupons from each growth protocol. The coupons can be made from a variety of materials, as desired. Biofilm growth can be controlled with specific changes in the growth medium, temperature, flow rate, rotation speed of the baffled stir bar and other growth conditions. The CDC reactor is available from BioSurfaces Technologies. $\mathbf{b}: \mathrm{A}$ biofilm growth setup using the $C D C$ reactor. A typical experiment would start with inoculation of the sterile reactor containing a nutrient solution and a period of batch operation with the effluent port clamped. Following the batch phase, the effluent port would be unclamped and a flow of a sterile fresh nutrient solution from the carboy, seen on the left, would start for a phase of continuous flow operation. A glass flow break in the feed line before the reactor prevents back-contamination of the feed carboy and maintains the influent sterility. A detailed species-specific growth protocol is described in the text. [The color figure for this article is available online at www. interscience. wiley.com.] 
fields due to motors and heaters switching on and off render it virtually useless for controlled magnetic field experiments. Therefore, a totally non-magnetic enclosure for the exposure setup was designed in which the temperature could be set and maintained. Since a uniform applied magnetic field was needed throughout a large volume, either a solenoid or a pair of Helmholtz coils had to be designed to apply the magnetic fields. A solenoid was selected since it could be designed to accommodate multiple laboratory scale biofilms and conditions during initial experimentation and also accommodate a full-scale prosthetic knee joint in future work. Finally, a treatment unit was designed that would allow the magnetic field exposure of biofilms while immersed in support medium. The top unit described below was designed to be used in conjunction with the CDC Biofilm Reactor so that biofilms would first be grown in the CDC Reactor and then aseptically moved to the individual compartments of the top unit during exposure.

\section{Non-Magnetic Environmental Box}

An economical, easy-to-build, non-magnetic environmental box is shown in Figure 2c. The frame of the box is made from $25.4 \mathrm{~mm}^{2}$ "snap together" aluminum tubes (manufactured by $80 / 20$, Columbia City, IN) with the top, sides, bottom and door made with $50.8 \mathrm{~mm}$-thick, closed cell Styrofoam insulation. The dimensions of the environmental box were chosen to fit a typical chemistry laboratory bench top and to accommodate the solenoid, top unit, pedestal and base plate (see Fig. 2a,b) in addition to control biofilms not exposed to the magnetic field. A small heating pad (seen at the back of the chamber and behind the solenoid in Fig. 2c) is used to hold the temperature in the chamber at $37^{\circ} \mathrm{C} \pm 1^{\circ}$. The $60 \mathrm{~Hz}$ magnetic field generated by the heating pad was measured using a digital magnetometer (Schonstedt Instrument, DM 2220-S5, Kearneysville, WV) and a oscilloscope (Tektronix, 2201, Wilsonville, $\mathrm{OR}$ ) and established at less than $6 \mathrm{mG}$ peak-to-peak at the level of the biofilm-coated coupons.

\section{Solenoid}

The design parameters for the solenoid included making the length at least three times the radius (to achieve a uniform magnetic field volume inside the solenoid), having an inside diameter large enough to allow the placement of a full-size prosthetic knee inside the coil, and having a coil form that was something "off-the-shelf." In order to minimize skin effect in the coil wires if frequencies above several hundred $\mathrm{Hz}$ were to be used, the wire chosen for the solenoid coils was Litzendraht (Litz) wire (New England Wire
Technologies, Type 2, \# 12, $5 \times 5 \times 42 / 42$, Lisbon, $\mathrm{NH}$ ). The Litz wire is manufactured using number 42 AWG insulated wire with five bundles of five wires each (all bundles are individually twisted) with 42 bundles twisted to form the final 1050 strand wire (with an outside diameter, including insulation, of $3 \mathrm{~mm}$ ). The coil form needed to have a wall thickness sufficient to allow the machining of spiral grooves to properly space three separate, non-overlapping coils of 47 turns wrapped on the form. The use of three windings was incorporated in the design of the solenoid in order to have flexibility in setting up the magnetic field and in the choice of the drive current in the coils. For example, one coil can be used to add to or zero-out the geomagnetic field, all three coils can be used in series to achieve a higher magnetic field at a given drive current, or one coil can be used to apply an AC field and two coils can be used to apply a DC field. Standard $203.2 \mathrm{~mm}$ (8-inch ID) PVC drain pipe with a wall thickness of approximately $9.5 \mathrm{~mm}$ was selected for the form as it is readily available and is easy to machine. The $660 \mathrm{~mm}$-long solenoid coil form with the exposure setup inside is shown in Figure $2 b$ and can be seen in Figure $2 \mathrm{c}$, with the coil wires in place and connected to the terminal strip bonded to the top of the coil form.

\section{Top Unit}

During treatment, the biofilm-coated coupons are aseptically transferred from the CDC biofilm reactor via the holders to the individual glass cylinders of the top unit (Fig. 2a). The top unit is then placed in the solenoid, sitting on the pedestal as seen in Figure 2b. Six of the coupon holders are arranged on ring $(r=60 \mathrm{~mm}$, $\varphi=n \times 60^{\circ}$; where $\left.n=0, \ldots, 5\right)$ with one holder centered along the $z$-axis (the coordinate system is shown in Fig. 2b). Each coupon holder holds three coupons and is contained in an individual glass cylinder (see Fig. 2a), $181 \mathrm{~mm}$ in height with an outer diameter of $50.8 \mathrm{~mm}$ (Technical Glass Products, part number RPMUSB50-3.5-7.125, Painesville, Twp., OH), which allows samples to be exposed in triplicate to each solution during magnetic field exposure. The $r=60 \mathrm{~mm}$ radius was selected for the ring of coupon holders since this is approximately the radius needed to achieve the closest packing of the six glass cylinders and to have them within the uniform magnetic field volume of the solenoid. A casing for the Schonstedt magnetometer probe was designed to be the same length and diameter as the coupon holders, so the casing and probe could be inserted in place of a coupon holder and magnetic field data could be obtained at each position. The rubber gasket seen in Figure 2a on the underside of the top plate prevents any cross-contamination between the glass treatment cylinders during the experiment and the entire 
a
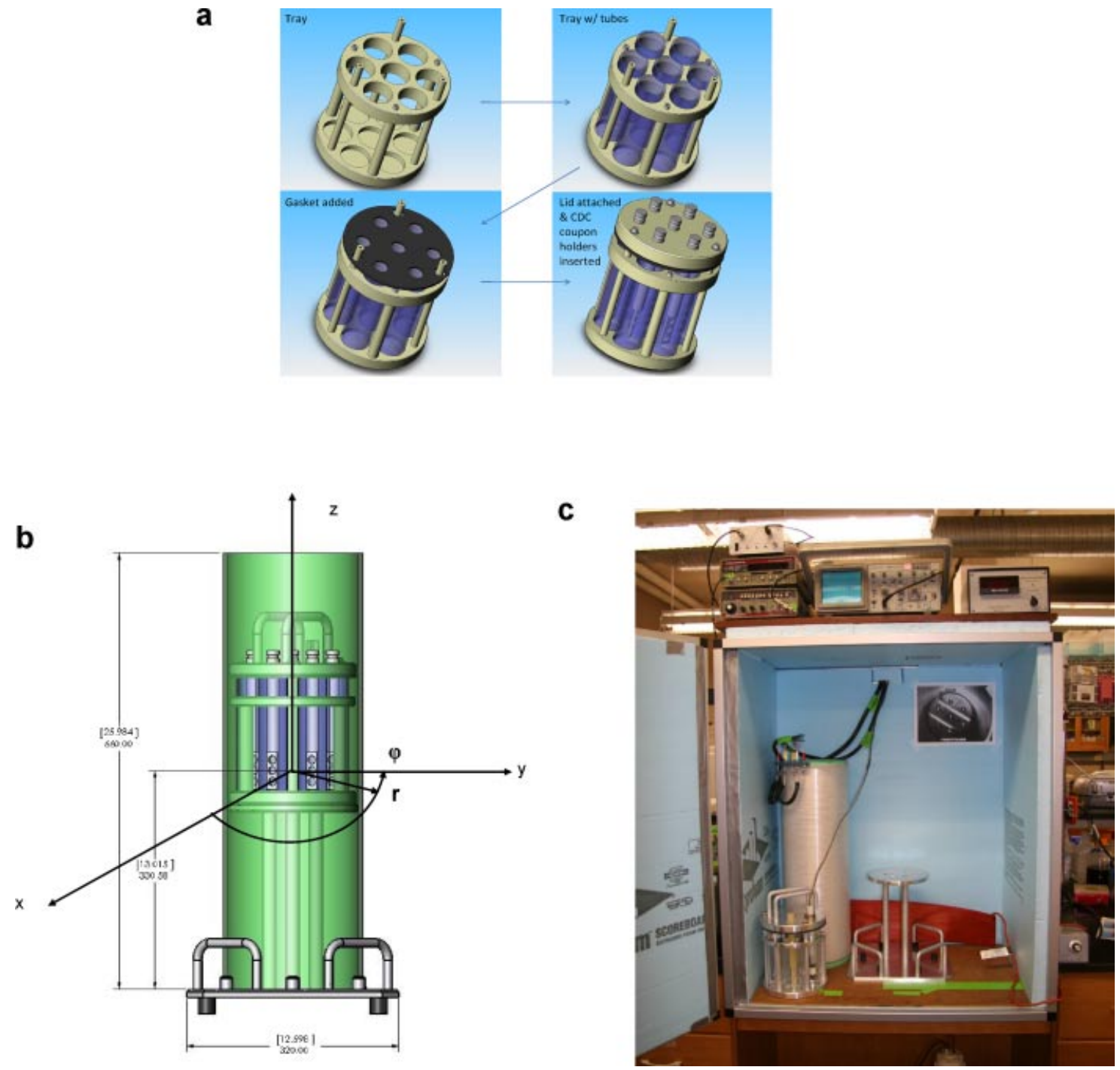

Fig. 2. The magnetic field exposure setup to be used during treatment. a: Four views of the top unit showing the tray, the tray with seven glass tubes inserted, the rubber gasket in place and the assembled top unit, including the lid with seven CDC polypropylene coupon holders projecting through the lid and into the glass tubes. $\mathbf{b}$ : A view of the top unit sitting on the pedestal inside of the solenoid coil form and the coordinate system. The handles used to lift the top unit in and out of the solenoid are shown in place on the top unit and coupons upon which the biofilms are grown are shown near the center of the solenoid coil form in place in the top unit. c: The non-magnetic bench top environmental chamber is seen with the door open to show the exposure setup top unit, pedestal, solenoid and heating pad. All electrical connections pass through the small port at the top, center of the chamber. The magnetometer probe is shown in place in the top unit on the $r=60 \mathrm{~mm}$ ring of coupon holders but it can be placed in any one of the seven positions. The N4L Model LPA01 power amplifier, the Circuitmate UC 10 Universal Frequency Counter (Beckman Instruments), the Circuitmate FG2 Function Generator (AC/DC power supply), the Tektronix 2201 Digital Storage Oscilloscope, and the Schoenstedt Instrument, Model 2220-S5 magnetometer are shown (top to bottom, left to right) in place on top of the chamber. [The color figure for this article is available online at www. interscience. wiley.com.]

top unit can be autoclaved. It should be noted that the coupon holders shown will allow the magnetic field to be applied parallel to the surface containing the biofilm. A second set of coupon holders are planned that rotate the surface of the coupons by $90^{\circ}$ making the biofilm surface perpendicular to the magnetic field (i.e., the $z$-axis).

The pedestal and base plate were designed to support the top unit and vertically locate the center coupon of the three biofilm coupons in the $z=0$ plane of 
the solenoid. The height of the environmental box was chosen to allow the top unit to be moved in and out of the solenoid when preparing an experimental exposure.

\section{MEASUREMENTS}

Figure 3 illustrates the measured and calculated magnetic fields, both inside and outside the solenoid. The calculated values for the magnetic field along the $z$-axis were done using the Biot-Savart law [Hayt, 1989] and for the magnetic fields off the $z$-axis, special expressions for the radial and $\mathrm{z}$ components of the magnetic field were used [Stratton, 1941]. The data outside the solenoid were developed in order to ascertain how non-exposed control biofilms could be positioned in the environmental box in order to receive a minimum amount of the magnetic field generated by the solenoid. Comparing data at a DC drive current of

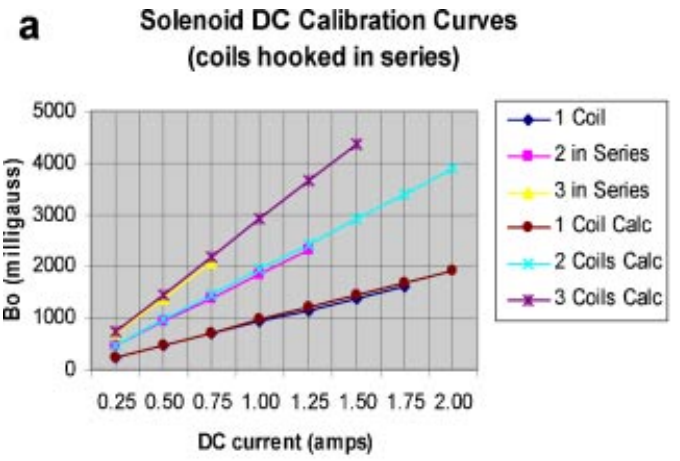

$0.6 \mathrm{~A}$, the magnetic field inside the solenoid is around $1700 \mathrm{mG}$ at $(0,0,0)$ while outside the coil at $(468 \mathrm{~mm}, 0,0)$ the field is approximately $17 \mathrm{mG}$. The scale (radius values) in Figure $3 c$ resulted from the method used to measure the magnetic field outside the solenoid. The pedestal and top unit were used outside of the solenoid to position the magnetometer probe in the same vertical plane as the internal probe placement ( $z=0$ plane) in order to measure the decrease of the external magnetic field generated by the solenoid with distance from the solenoid. Due to the width of the pedestal base, the closest position of the probe to the solenoid was $268 \mathrm{~mm}$ and each data point (starting from $268 \mathrm{~mm}$ ) is, thereafter, a change in the $r$-value of $20 \mathrm{~mm}$.

One of the design goals for the solenoid was to be able to apply both low level and high level AC magnetic fields, from frequencies below $100 \mathrm{~Hz}$ to frequencies as
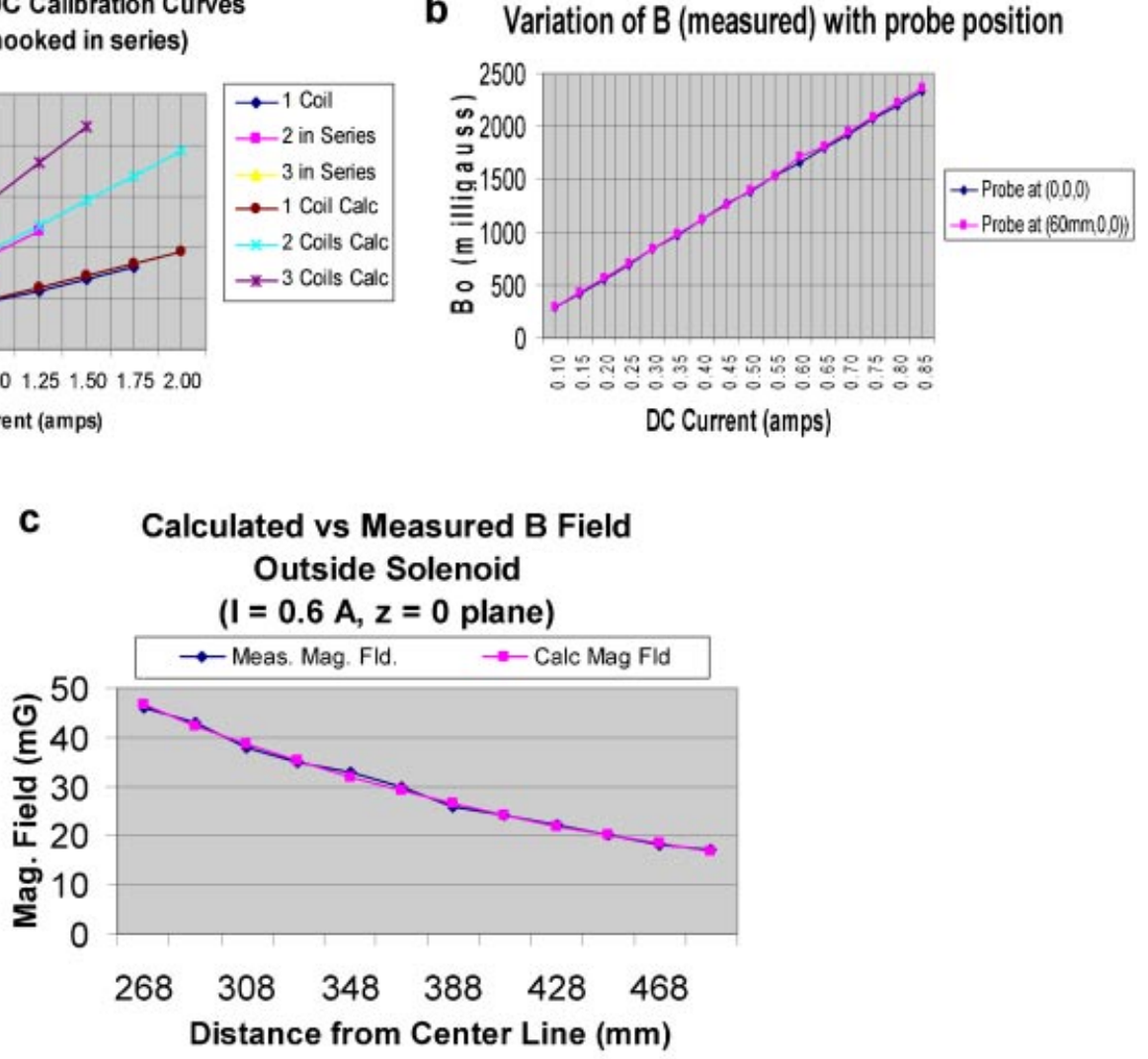

Fig. 3. Chart (a) shows both calculated and measured values at $(0,0,0)$ for a single coil, two coils in series (fields adding) and three coils in series (fields adding) versus the DC drive current. All magnetic field measurements were done with a single Schonstedt Instrument DM 2220-S5 digital magnetometer. Chart (b) shows the measured magnetic fields versus DC current with three coils in series at the origin $(0,0,0)$ and at one of the positions on the outer ring $(60 \mathrm{~mm}, 0,0)$. Differences are negligible out to at least $60 \%$ of the radius of the solenoid. Chart (c) compares calculated and measured values for the magnetic field outside the solenoid and in the $z=0$ plane for $(r)$ between $268 \mathrm{~mm}$ (closest to the solenoid) and $488 \mathrm{~mm}$ (near the right side wall of the environmental chamber) again using a 0.6 A DC drive current. [The color figure for this article is available online at www. interscience. wiley.com.] 
high as $1 \mathrm{kHz}$. As noted above, the wire selected for the solenoid was type $2 \mathrm{Litz}$ wire which has an operating frequency range up to $350 \mathrm{kHz}$ and a current capacity of about $5 \mathrm{~A}$. Laboratory measurements with the three coils in series show an AC magnetic field (sine wave) of 3.2 gauss (peak-to-peak) can be achieved with a frequency of $100 \mathrm{~Hz}$, and 0.46 gauss (peak-to-peak) can be achieved at $1 \mathrm{kHz}$. These data were obtained using a Circuitmate FG2 function generator (Beckman Instruments, Fullerton, CA) in series with an N4L model LPA01 laboratory power amplifier (Newtons4th, Arlington Heights, IL) to drive the coils.

The data in Figure 3 show that the magnetic fields inside the solenoid at $(0,0,0)$ are in close agreement with the fields at $(60 \mathrm{~mm}, 0,0)$ as expected (see Fig. 3b) and the field is essentially constant across this radius. Measurements and calculations (not shown) also indicate the magnetic field variation along the $z$-axis (vertical) of the solenoid was less than $1 \%$ over the dimension of the biofilm coupons and less than $5 \%$ for $z= \pm 150 \mathrm{~mm}$. Therefore, the constant, cylindrical magnetic field volume is at least $60 \mathrm{~mm}$ in radius, with a height of at least $z= \pm 150 \mathrm{~mm}$.

\section{CONCLUSIONS}

This article focused on two points. First, a wellcharacterized, repeatable and "exportable" method of growing bacterial biofilms on material surfaces is extremely important when doing any biofilm work. Biofilms can be grown on various surfaces by a number of means, but growing "laboratory grade" biofilms can prove to be a challenge. The growth protocol described was optimized for S. epidermidis ATCC\#35984 grown in the CDC Reactor and the resulting biofilms show a high level of repeatability. Secondly, an exposure setup was described that was designed to study how magnetic fields might be used alone, or in conjunction with antibiotics, to control bacterial biofilms on materials typically found in prosthetic implants. Since bacterial biofilm experiments often require a controlled and elevated temperature (in this case, body temperature) and since laboratory incubators incorporate so many uncontrollable magnetic fields, one design of a nonmagnetic environmental chamber is described. The exposure setup presented was specifically designed to be compatible with the CDC Biofilm Reactor and to allow the testing of up to seven different solutions with varying levels of antibiotics, nutrient content, trace metals, etc., in each experimental run. This system will aid in the study of magnetic fields applied to biofilms, which are found on orthopedic implants. The system is capable of applying magnetic fields, both DC and AC fields (up to about $1 \mathrm{kHz}$ ), from ten to several thousand milligauss. Additionally, the system can be modified for different bacterial species and because the coupons in the CDC reactor can be made of a variety of materials, the system is sufficiently flexible to allow it to be useful for the study of other applications.

\section{ACKNOWLEDGMENTS}

The artwork and manufacturing design drawings for the solenoid and experimental exposure setup were done by Mr. Joe Eldring, Manager, Technical Services, Montana State University College of Engineering.

\section{REFERENCES}

Aaron R, Ciombor D, Simon B. 2004. Treatment of nonunions with electric and electromagnetic fields. Clin Orthop Relat Res 419:21-29.

ASTM. 2007. ASTM Standard E 5895-07. "Quantification of Pseudomonas aeruginosa Biofilm Grown with High Shear and Continuous Flow using CDC Biofilm Reactor". West Conshohocken, PA: ASTM International. 10.1520/E256207. www.astm.org.

Blank M. 1993. Electricity and magnetism in biology and medicine: Review and research papers presented at the First World Congress for Electricity and Magnetism in Biology and Medicine, Orlando, Florida, 1992. San Francisco, CA, USA: San Francisco Press. xv, 936 pp.

Blenkinsopp S, Khoury A, Costerton J. 1992. Electrical enhancement of biocide efficacy against Pseudomonas aeruginosa biofilms. Appl Environ Microbiol 58(11):3770-3773.

Buckingham-Meyer K, Goeres D, Hamilton M. 2007. Comparative evaluation of biofilm disinfectant efficacy tests. J Microbiol Methods 70(2):236-244.

Caubet R, Pedarros-Caubet F, Chu M, Freye E, de Belém Rodrigues M, Moreau J, Ellison W. 2004. A radio frequency electric current enhances antibiotic efficacy against bacterial biofilms. Antimicrob Agents Chemother 48(12):4662-4664.

Costerton JW, Stewart PS. Biofilms and device-related infections. In: Nataro JP, Blaser MJ, Cunningham-Rundles S, editors. 2000. Persistent bacterial infections. Washington, DC: ASM Press. xv, 453, pp. 423-439.

Darouiche R. 2004. Treatment of infections associated with surgical implants. N Engl J Med 350(14):1422-1429.

Del Pozo J, Rouse M, Patel R. 2008. Bioelectric effect and bacterial biofilms. A systematic review. Int J Artif Organs 31(9):786795.

Ehrlich G, Stoodley P, Kathju S, Zhao Y, McLeod B, Balaban N, Hu F, Sotereanos N, Costerton J, Stewart P. 2005. Engineering approaches for the detection and control of orthopaedic biofilm infections. Clin Orthop Relat Res 437:59-66.

Giladi M, Porat Y, Blatt A, Wasserman Y, Kirson E, Dekel E, Palti Y. 2008. Microbial growth inhibition by alternating electric fields. Antimicrob Agents Chemother 52(10):3517-3522.

Goeres D, Loetterle L, Hamilton M, Murga R, Kirby D, Donlan R. 2005. Statistical assessment of a laboratory method for growing biofilms. Microbiology 151(Pt 3):757-762.

Hayt WH. 1989. Engineering electromagnetics. New York: McGraw-Hill. xiii, 472 pp.

König D, Schierholz J, Münnich U, Rütt J. 2001. Treatment of staphylococcal implant infection with rifampicin- 
ciprofloxacin in stable implants. Arch Orthop Trauma Surg 121(5):297-299.

Masterson E, Masri B, Duncan C. 1998. Treatment of infection at the site of total hip replacement. Instr Course Lect 47:297-306.

O'Gara J, Humphreys H. 2001. Staphylococcus epidermidis biofilms: Importance and implications. J Med Microbiol 50(7):582-587.

Pavoni G, Giannella M, Falcone M, Scorzolini L, Liberatore M, Carlesimo B, Serra P, Venditti M. 2004. Conservative medical therapy of prosthetic joint infections: Retrospective analysis of an 8-year experience. Clin Microbiol Infect 10(9): 831-837.

Pilla L, Valenti R, Marrari A, Patuzzo R, Santinami M, Parmiani G, Rivoltini L. 2006. Vaccination: Role in metastatic melanoma. Expert Rev Anticancer Ther 6(8):1305-1318.

Saginur R, Stdenis M, Ferris W, Aaron S, Chan F, Lee C, Ramotar K. 2006. Multiple combination bactericidal testing of staphylococcal biofilms from implant-associated infections. Antimicrob Agents Chemother 50(1):55-61.
Shirtliff M, Bargmeyer A, Camper A. 2005. Assessment of the ability of the bioelectric effect to eliminate mixedspecies biofilms. Appl Environ Microbiol 71(10):63796382.

Stewart P, Wattanakaroon W, Goodrum L, Fortun S, McLeod B. 1999. Electrolytic generation of oxygen partially explains electrical enhancement of tobramycin efficacy against Pseudomonas aeruginosa biofilm. Antimicrob Agents Chemother 43(2):292-296.

Stratton JA. 1941. Electromagnetic theory. New York, London, McGraw-Hill Book Company, Inc. xv, 615 pp.

Wellman N, Fortun S, McLeod B. 1996. Bacterial biofilms and the bioelectric effect. Antimicrob Agents Chemother 40(9): 2012-2014.

Zimmerli W, Widmer A, Blatter M, Frei R, Ochsner P. 1998. Role of rifampin for treatment of orthopedic implant-related staphylococcal infections: A randomized controlled trial. Foreign-Body Infection (FBI) Study Group. JAMA 279(19): 1537-1541. 